\title{
Chunnel vision
}

\section{Export and efflux through bacterial channel-tunnels}

\author{
Christian Andersen, Colin Hughes and Vassilis Koronakis ${ }^{+}$
}

University of Cambridge Department of Pathology, Tennis Court Road, Cambridge CB2 1QP, UK

Received August 1, 2000; revised August 27, 2000; accepted August 28, 2000

The Escherichia coli TolC protein is central to toxin export and drug efflux across the inner and outer cell membranes and the intervening periplasmic space. The crystal structure has revealed that TolC assembles into a remarkable $\alpha$-helical trans-periplasmic cylinder (tunnel) embedded in the outer membrane by a contiguous $\beta$-barrel (channel), so providing a large duct open to the outside environment. The channeltunnel structure is conserved in TolC homologues throughout Gram-negative bacteria, and it is envisaged that they are recruited and opened, through a common mechanism, by substrate-specific inner-membrane complexes.

\section{Export and efflux across the periplasmic space}

In Gram-negative bacteria, proteins destined for the cell surface or the surrounding medium must cross the cytoplasmic (inner) and outer membranes, and the periplasm between them. Several pathways achieve this by two-step mechanisms that employ periplasmic intermediates [the type II general secretion pathway (Russel, 1998) and adhesion pilus assembly (Hultgren et al., 1993)], or by using a large number of proteins in machineries spanning the envelope (the type III assembly of flagella and invasomes; Hueck, 1998). The type I export mechanism contrasts with these, as it requires only three proteins and does not generate periplasmic intermediates (Koronakis et al., 1989, 1991). It exports many large proteins, including acylated toxins like the $110 \mathrm{kDa}$ Escherichia coli hemolysin and $170 \mathrm{kDa}$ Bordetella pertussis cyclolysin, and the 60-80 kDa proteases of Serratia marcescens and Pseudomonas aeruginosa (Glaser et al., 1988; Koronakis and Hughes, 1993; Paulsen et al., 1997). The three export components are a traffic ATPase and an accessory protein, which together form an energized substrate-specific complex in the inner membrane (IM), and a third protein, a member of the TolC family, in the outer membrane (OM)
(Koronakis and Hughes, 1993; Paulsen et al., 1997). A closely related mechanism effects efflux of small noxious compounds such as detergents, organic solvents, ethidium bromide and antibacterial drugs such as nalidixic acid and antibiotics like tetracycline, erythromycin and chloramphenicol (Zgurskaya and Nikaido, 2000). Analogous to the type I protein export machineries, these 'efflux pumps' comprise an OM TolC-family protein cooperating with a substrate-specific IM complex. However, in this case the closely related accessory protein interacts in the IM complex with a protein providing energy from proton antiport, not ATP hydrolysis (Zgurskaya and Nikaido, 2000). TolC and its homologues clearly have an important role in bacterial survival, and notably confer virulence and clinically important multidrug resistance (MDR) during infection of human and animal hosts.

TolC family members are widespread among Gram-negative bacteria. Figure 1 depicts those with known or putative function, arranged as a phylogenetic tree based on amino acid sequence relatedness. It has been observed that the sequences of the $\mathrm{N}$ and C-terminal halves of the proteins are similar to each other (Johnson and Church, 1999), indicating that the family has evolved by a gene duplication. We note that the strongest identity between the two halves $(29.1 \%)$ is evident in B. pertussis CyaE, which is involved in cyclolysin export, and it is also nearest to the root of the family tree, suggesting that it is closest to the family progenitor. Sequence similarity among the TolC protein family correlates with exporter substrate specificity, indeed the proteins can be grouped into three subfamilies corresponding to their roles in protein export, cation efflux and drug efflux. While E. coli TolC exemplifies the protein export subfamily, combining with IM complexes energized by traffic ATPases, the homologues of the drug- and cation-efflux subfamilies typically cooperate with the RND (resistance/ nodulation/division) family of IM transport complexes. TolC homologues and their IM partners are usually encoded within the same operons, e.g. the $B$. pertussis cyclolysin operon

+Corresponding author. Tel: +44 1223 333740; Fax: +44 1223 333346; E-mail: vk103@mole.bio.cam.ac.uk 


\section{review}

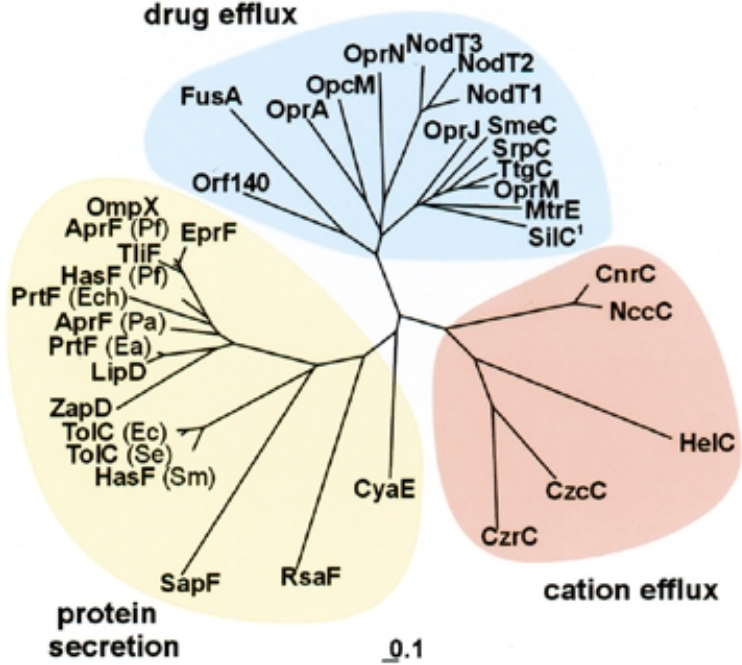

Fig. 1. Phylogenetic tree of the TolC family. The 36 TolC homologues for which function is known, or strongly implicated by the location of their genes in the export or efflux operons, sorted by TREEVIEW (Page, 1996) on the basis of a sequence alignment using MULTIALIN (Corpet, 1988). Abbreviations: Ea, Erwinia amylovora; Ec, Escherichia coli; Ech, Erwinia chrysanthemi; Se, Salmonella enteritidis; Sm, Serratia marcescens; Pa, Pseudomonas aeruginosa; Pf, Pseudomonas fluorescens. SilC ${ }^{1}$ may be an exception to the subgrouping as it is reported to be involved in silver efflux. Scale ' 0.1 ' indicates 0.1 nucleotide substitutions. Note: the database has a further 20 homologues, with no ascribed function to date.

cyaCABDE and the $P$. aeruginosa MDR operon mexABoprM (Glaser et al., 1988; Zgurskaya and Nikaido, 2000). To date, E. coli TolC is an exception, as it is not encoded by an export/ efflux operon (it is suggested to be part of the stress-induced mar-sox regulon; Aono et al., 1998). Furthermore, it acts with multiple IM partners to effect both protein export and drug efflux, specifically with translocases containing the traffic ATPases HlyB and CvaA, the AcrA/B complex of the RND family and putatively the EmrA/B complex of the major facilitator superfamily (MFS) (Koronakis and Hughes, 1993; Paulsen et al., 1997; Zgurskaya and Nikaido, 2000).

The underlying mechanism has been studied in the TolCdependent hemolysin export by $E$. coli. TolC is clearly an integral part of the export machinery. When TolC is deleted there is no residual export to the periplasm or from spheroplasts (Koronakis et al., 1997), and cross-linking has shown that TolC is recruited by the IM translocase in response to engagement of the protein substrate at the cytoplasmic membrane (Thanabalu et al., 1998). Contact between the IM complex and TolC is mediated principally by the translocase accessory protein, and the active type I export complex contains the substrate and all three export proteins, each of which undergoes conformational change. This complex is transient; once the substrate passes out of the cell the IM and OM components disengage and revert to their resting states. However, it was still unclear how the IM complexes could connect with a TolC homologue to bridge the periplasm, believed to measure 130-140 $\AA$ across (Graham et al., 1991), especially if TolC had a simple OM porin-like structure. It had been widely thought that the bridging role would be fulfilled by the family of accessory proteins in the IM complexes that had been named the 'membrane fusion protein' family (Dinh et al., 1994) and variously proposed to span the periplasm or bring the inner and outer membranes into close apposition (Dinh et al., 1994; Paulsen et al., 1997; Johnson and Church, 1999; Zgurskaya and Nikaido, 2000). The first indication that TolC might be unconventional, and possibly contribute to the periplasmic bypass, was provided by electron microscopy of TolC reconstituted into lipid bilayers. This analysis of twodimensional crystals showed that TolC was a trimeric structure, indeed reminiscent of porins. However, it also hinted at a single pore rather than three, and the possible existence of a periplasmic domain (Koronakis et al., 1997). While prescient, these observations proved scant preparation for what the high resolution three-dimensional structure would uncover.

\section{A channel-tunnel spanning the periplasmic space}

X-ray crystallography of the TolC protein has recently revealed a remarkable structure, described in detail by Koronakis et al. (2000). Seen at $2.1 \AA$ resolution, a TolC homotrimer forms a hollow tapered cylinder $140 \AA$ in length that comprises a $100 \AA$ long $\alpha$-helical barrel (the tunnel domain) anchored in the OM by a $40 \AA$ long $\beta$-barrel (the channel domain). A mixed $\alpha / \beta$ structure, the equatorial domain, forms a 'strap' around the mid section of the helical barrel, facing outwards. The diameter of this conduit is $35 \AA$, forming a water-filled $43000 \AA^{3}$ duct open to the cell exterior. Towards the periplasmic end, the inner diameter decreases to a virtual close (Figure 2).

Although an embedded $\beta$-barrel is a feature of other OM proteins, the TolC $\beta$-barrel is quite distinct in its architecture. All other known OM proteins form one barrel per protein monomer (Figure 2). Of the monomeric proteins, OmpA forms the smallest $\beta$-barrel of eight $\beta$-strands, resulting in a diameter of $26 \AA$ (Pautsch and Schulz, 1998), while the largest $\beta$-barrels, found in the iron siderophore transporters FhuA and FepA (Locher et al., 1998; Buchanan et al., 1999), comprise $22 \beta$-strands and determine an elliptical diameter of 39-46 $\AA$. The OM phospholipase A (OMPLA) is active as a dimer of $12 \beta$-strand barrels (Snijder et al., 1999). The widespread channel-forming porins are trimeric structures, in which each monomer forms a $\beta$-barrel of 16 (e.g. OmpF) or 18 (e.g. LamB) $\beta$-strands (Cowan et al., 1992; Schirmer et al., 1995). In TolC, the three molecules each contribute four $\beta$-strands to form a single 12 strand $\beta$-barrel. This architecture is not known in cell membrane proteins, but is seen in the pore-forming Staphylococcus aureus $\alpha$-toxin, which has seven subunits forming a barrel of $14 \beta$-strands (Song et al., 1996). The interior of the TolC channel is also different from those of reported proteins (Figure 2). The cross-sectional area of the channel is $960 \AA^{2}$, making it 15-fold larger than that of the general diffusion pore OmpF, which allows passage of molecules up to 600 Da (Cowan et al., 1992). It also contrasts markedly with the $\beta$-barrels of OmpA and OMPLA, the diameters of which are too small to allow the passage of ions. TolC lacks the common structural element of channel-forming proteins, an inward folded loop that constricts the internal diameter of the $\beta$-barrel and has a strong influence on channel properties (Jordy et al., 1996; Saint et al., 1996). It also differs 


\section{review}

Export and efflux through bacterial channel-tunnels

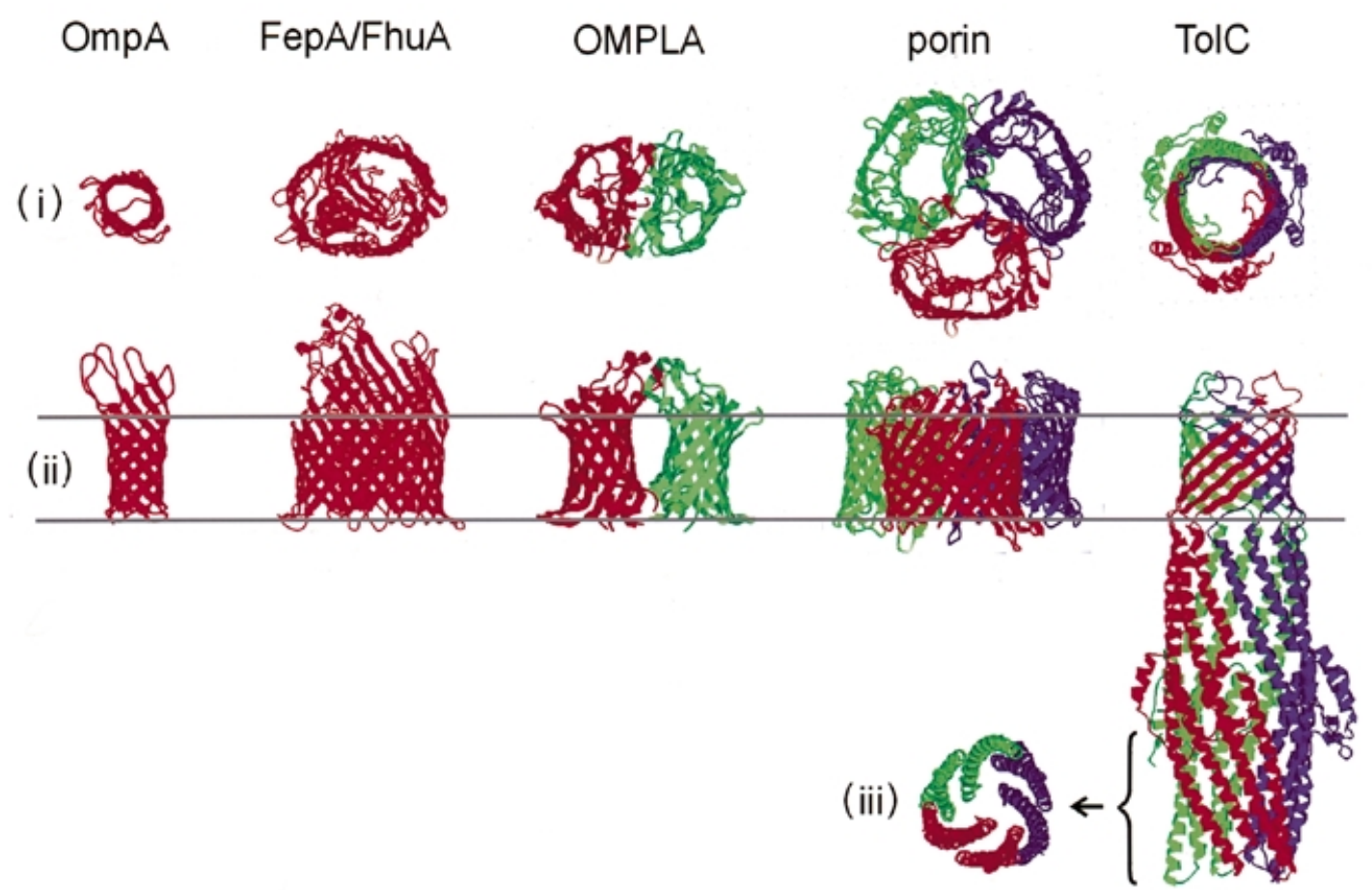

Fig. 2. The structures of bacterial OM proteins. (i) Top view of the proteins down the OM channel. In the case of TolC this extends to $\sim 2 / 3$ of the height, i.e. the top $100 \AA$ A. (ii) Side view, at right angles to the plane of the OM. (iii) Cross-section of TolC near the tunnel entrance, i.e. the bottom $40 \AA$ A. Red, green and blue indicate individual monomers.

from that of the larger $\beta$-barrels of the FhuA and FepA iron transporters, which are both closed by plug domains. Nevertheless, electrophysiological measurements show that TolC is a channelforming protein, albeit of only low conductance (Benz et al., 1993; C. Andersen and V. Koronakis, unpublished), presumably due to the closed periplasmic entrance. Notwithstanding these singularities of the TolC $\beta$-barrel, the most striking difference from other known OM proteins is the $100 \AA$ long periplasmic tunnel (Figure 2), comprising a previously unknown fold, a 12 strand $\alpha$-helical barrel. Like the $\beta$-barrel channel, the tunnel is assembled from four antiparallel strands per monomer (two continuous long helices and two pairs of shorter helices). It thus generates a continuous proteinaceous duct for the exit of substrates bound specifically at the IM.

\section{Conservation of the channel-tunnel structure}

The $\mathrm{N}$ - and C-terminal halves of the 471 residue TolC protein can be structurally superimposed (Koronakis et al., 2000), despite having only moderate sequence similarity (34.5\%). This structural duplication is evident throughout the family. Sequences encoding the $\alpha$-helices and $\beta$-strands of the channeltunnel structure do not vary substantially in length among the homologues: the long $\alpha$ - helices are constant at 67 residues and the shorter helices are 23 and 34 residues. This suggests that the basic fold of the structure is conserved. While the 56 reported TolC homologues vary in length from 414 to 541 amino acids, this is due primarily to variable extensions at the periplasmic $\mathrm{N}$ - and C-termini [32-96 amino acids (unprocessed) and 1-99 amino acids, respectively], with the multidrug efflux subclass having a characteristic acylation site in the extended $\mathrm{N}$-terminus. Significant sequence gaps or insertions occur only in the mixed $\alpha / \beta$ equatorial domain, which is not part of the $\alpha / \beta$ barrel structure, and in the extracellular loops. The loops are characteristically very small in homologues involved in cation efflux.

Only a small number of amino acids are common to all known homologues (Figure 3), and these and others that are well conserved correlate with features that determine the core structure. At or near the tunnel entrance, glycine residues facilitate a tight turn between the helices forming the entrance itself. Small residues, such as alanine and serine, at the interface of tunnelforming helices allow a very dense packing that determines the tapering and closure, and aspartic acid residues maintain the electronegative inner surface near the entrance, which may influence the movement of substrate. Towards the other end of the structure, the transition from the left-handed $\alpha$-helices of the periplasmic domain to the right-handed $\beta$-strands of the outer membrane domain is accommodated by proline-containing linkers (except that for one of the four strands there is divergence in the efflux subfamilies, with glycines mixed in with, or replacing prolines). At the bottom of the $\beta$-strands, aromatic residues face outwards to form a ring around the channel domain, delimiting the inner edge of the lipid bilayer. This is seen in all 


\section{review}

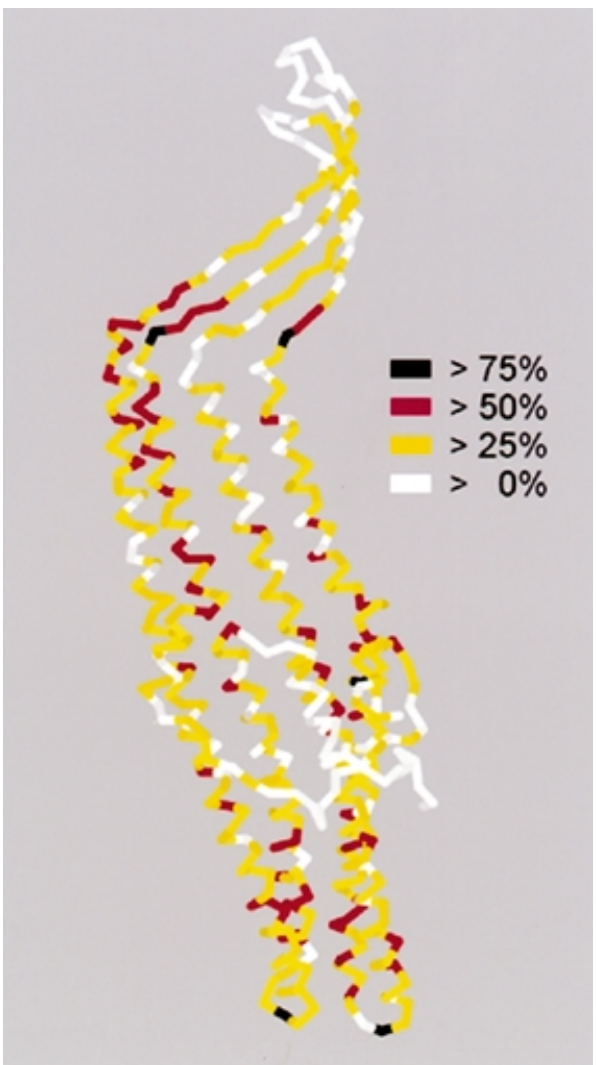

Fig. 3. Sequence conservation of TolC homologues related to the TolC structure. The TolC monomer coloured, as indicated, according to the level of consensus of individual amino acids throughout the known 56 TolC homologues. Alignment was performed using MULTIALIN (Corpet, 1988), consensus scores were calculated using the CLUSTAL function with matrix PAM350.

crystallized OM proteins and possibly has an anchor function. Of less obvious functional significance is the leucine residue that is always found at the junction of the interrupted helix strands. Its importance may lie in the process or stabilization of folding. Overall, these comparisons between TolC family members confirm that the defining structural elements are conserved and strongly suggest that all homologues fold similarly and have comparable properties. One can reasonably conclude that the core functions of the channel-tunnel are common throughout Gram-negative bacteria.

\section{A common mechanism of export and efflux via channel-tunnels}

The elucidation of the structure of the TolC protein vividly answers the central question of how the periplasmic space is bypassed, and the conservation of the key structural amino acids among TolC homologues establishes a common mechanism for the export and efflux systems dependent upon this family of proteins. One can readily visualize that substrate-laden IM translocases recruit and open cognate channel-tunnels, providing gated exit of diverse molecules from the cell interior, through trans-periplasmic ducts to the external environment. Whether the TolC structure has relevance to other types of transport is an interesting question. The problem of protein import across two membranes into the mitochondrial matrix (Herrmann and Neupert, 2000) presents provocative parallels as it is mediated by reversible association between complexes in the outer and inner membranes, and does not involve intermediates in the intermembrane space. As mitochondria are generally believed to have evolved from bacteria, it is possible that mitochondrial import machines and the type I exporters of Gram-negative prokaryotes might have common ancestors. Tim23, a key component of the mitochondrial preprotein translocase, is anchored by its C-terminal domain to the inner mitochondrial membrane, spans the inter-membrane space and penetrates the outer membrane via predicted $\beta$-sheets in its $\mathrm{N}$-terminus (Donzeau et al., 2000). Tim23 is at least a dimer comprised of 222 amino acid monomers. Overall, this structure is similar in size to half of TolC. Is it possible that Tim23 corresponds to the unit of gene duplication in the prokaryotic proteins?

The principles by which TolC operates seem evident. A view of TolC from above the OM (i.e. the exit) down to the equatorial domain shows a uniform cylinder with an inner diameter of $35 \AA$ (Figure 2), through which even large proteins could readily pass. In contrast, the periplasmic entrance of TolC must undergo conformational change to allow passage of substrate. An allosteric mechanism has been proposed (Koronakis et al., 2000) whereby a realignment of the inner pair of helices of each monomer to that of the outer pair could open the entrance, like an iris, to a diameter of $30 \AA$. This opening would be triggered and stabilized by another component, evidently the recruiting IM complex in response to engagement by the export/efflux substrate. Cross-linking has shown that the principle contact between this substrate-bound IM complex and TolC is made by the accessory protein, and that like $\mathrm{TolC}$, this protein is trimeric (Thanabalu et al., 1998). A potential site for the key action of the accessory protein is the tunnel entrance itself. However, the accessory proteins are also highly likely to form coiled-coil structures of varying lengths (Johnson and Church, 1999), which could potentially repack against the coiled-coils of the tunnel $\alpha$-barrel and/or reach to the equatorial domain to effect opening of the entrance (Figure 4). As the accessory protein interacts with both the channel-tunnel and the energy-providing protein, it might act as a dynamic adaptor. When the protein secretion system contains a mutant traffic ATPase, and ATP is bound but not hydrolysed, substrate is not exported but instead accumulates in the assembled IM-OM export complex (Thanabalu et al., 1998). This suggests that, whereas substrate entry to the IM translocase might be gated by the binding of ATP and substrate, release of substrate into the recruited TolC could occur in a distinct step that is dependent upon ATP hydrolysis.

\section{Perspectives}

The mechanistic details of tunnel opening and interaction with cognate adaptors during export and efflux are now accessible. Knowledge of the chunnel structure also opens the way to defining the site of interaction (the external loops?) with bactericidal colicins that require TolC to enter the cell (Pilsl and Braun, 1995; Lazdunski et al., 1998). There remains the question of how and when the trimeric structure is assembled. Also, how is 


\section{review}

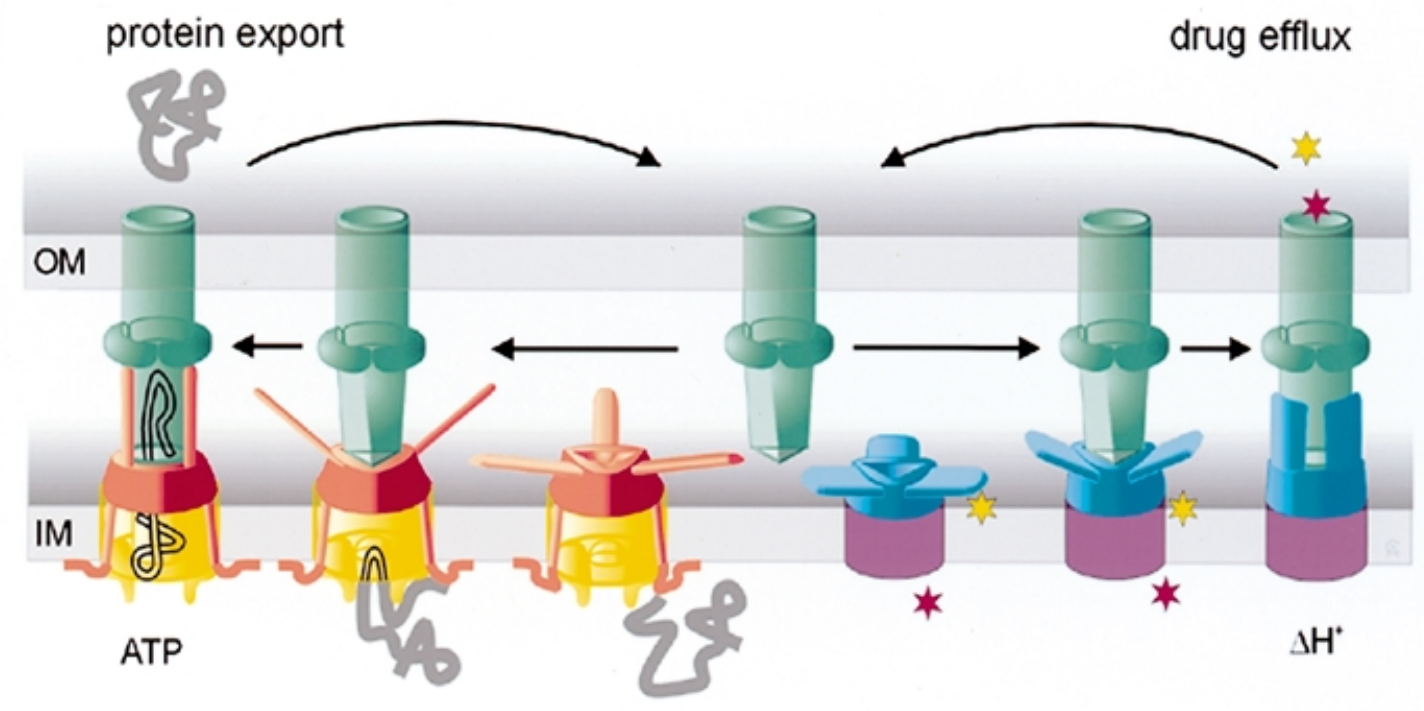

Fig. 4. Channel-tunnels of the TolC family acting in export and efflux. A model indicating reversible interaction of trimeric TolC homologues (green) with substrate-specific IM complexes containing an adaptor protein (red/blue) and an energy-providing protein of either the traffic ATPase (protein export, yellow) or proton antiporter families (small molecule efflux, purple). Escherichia coli TolC can act in both pathways, while in other bacteria multiple TolC homologues act separately in distinct pathways. In response to substrate engagement [a peptide chain, or hydrophilic and hydrophobic drugs (stars)], the trimeric adaptor protein contacts the periplasmic tunnel, possibly via the predicted coiled-coil structures indicated, prompting the conformational change that opens the entrance.

the peptidoglycan layer negotiated? Perhaps cellular enzymes that degrade the peptidoglycan might have a function in TolC assembly, analagous to the dedicated muraminidase needed for assembly of flagella on the cell surface (Nambu et al., 1999). Another question relates to how the channel-tunnels are located by substrate-laden IM complexes. Recruitment seems likely to be stochastic, but the resting state might already involve a loose interaction, too weak to be dedicated by cross-linking. Whatever the details, the startling and beautiful TolC structure is the latest in a stream of bacterial outer membrane protein structures that are setting a new agenda in our understanding of translocation processes.

\section{Acknowledgements}

We thank Eva Koronakis for helpful criticism of the manuscript. Our work is supported by a Medical Research Council Programme grant (V.K. and C.H.). C.A. is an EMBO fellow.

\section{References}

Aono, R., Tsukagoshi, N. and Yamamoto, M. (1998) Involvement of outer membrane protein TolC, a possible member of the mar-sox regulon, in maintenance and improvement of organic solvent tolerance of Escherichia coli K-12. J. Bacteriol., 180, 938-944.

Benz, R., Maier, E. and Gentschev, I. (1993) TolC of Escherichia coli functions as an outer membrane channel. Zentralbl. Bakteriol., 278, 187196.

Buchanan, S.K, Smith, B.S., Venkatramani, L., Xia, D., Esser, L., Palnitkar, M., Chakraborty, R., van der Helm, D. and Deisenhofer, J. (1999) Crystal structure of the outer membrane active transporter FepA from Escherichia coli. Nature Struct. Biol., 6, 56-63.
Corpet, F. (1988) Multiple sequence alignment with hierarchical clustering. Nucleic Acids Res., 16, 10881-10890.

Cowan, S.W., Schirmer, T., Rummel, G., Steiert, M., Ghosh, R., Pauptit, R.A., Jansonius, J.N. and Rosenbusch, J.P. (1992) Crystal structures explain functional properties of two E.coli porins. Nature, 358, 727-733.

Dinh, T., Paulsen, I.T. and Saier, M.H., Jr (1994) A family of extracytoplasmic proteins that allow transport of large molecules across the outer membranes of gram-negative bacteria. J. Bacteriol., 176, 3825-3831.

Donzeau, M., Kaldi, K., Adam, A., Paschen, S., Wanner, G., Guiard, B., Bauer, M.F., Neupert, W. and Brunner, M. (2000) Tim23 links the inner and outer mitochondrial membranes. Cell, 101, 401-412.

Glaser, P., Sakamoto, H., Bellalou, J., Ullmann, A. and Danchin, A. (1988) Secretion of cyclolysin, the calmodulin-sensitive adenylate cyclasehaemolysin bifunctional protein of Bordetella pertussis. EMBO J., 7, 3997-4004.

Graham, L.L., Harris, R., Villiger, W. and Beveridge, T.J. (1991) Freezesubstitution of gram-negative eubacteria: general cell morphology and envelope profiles. J. Bacteriol., 173, 1623-1633.

Herrmann, J.M. and Neupert, W. (2000) Protein transport into mitochondria. Curr. Opin. Microbiol., 3, 210-214.

Hueck, C.J. (1998) Type III protein secretion systems in bacterial pathogens of animals and plants. Microbiol. Mol. Biol. Rev., 62, 379-433.

Hultgren, S.J., Abraham, S., Caparon, M., Falk, P., St Geme, J.W., III and Normark, S. (1993) Pilus and nonpilus bacterial adhesins: assembly and function in cell recognition. Cell, 73, 887-901.

Johnson, J.M. and Church, G.M. (1999) Alignment and structure prediction of divergent protein families: periplasmic and outer membrane proteins of bacterial efflux pumps. J. Mol. Biol., 287, 695-715.

Jordy, M., Andersen, C., Schulein, K., Ferenci, T. and Benz, R. (1996) Rate constants of sugar transport through two LamB mutants of Escherichia coli: comparison with wild-type maltoporin and LamB of Salmonella typhimurium. J. Mol Biol., 259, 666-678.

Koronakis, V. and Hughes, C. (1993) Bacterial signal peptide-independent protein export: HlyB-directed secretion of hemolysin. Semin. Cell Biol., 4, $7-15$. 


\section{review}

\section{Andersen, C. Hughes and V. Koronakis}

Koronakis, V., Koronakis, E. and Hughes, C. (1989) Isolation and analysis of the C-terminal signal directing export of Escherichia coli hemolysin protein across both bacterial membranes. EMBO J., 8, 595-605.

Koronakis, V., Hughes, C. and Koronakis, E. (1991) Energetically distinct early and late stages of HlyB/HlyD-dependent secretion across both Escherichia coli membranes. EMBO J., 10, 3263-3272.

Koronakis, V., Li, J., Koronakis, E. and Stauffer, K. (1997) Structure of TolC, the outer membrane component of the bacterial type I efflux system, derived from two-dimensional crystals. Mol. Microbiol., 23, 617-626.

Koronakis, V., Sharff, A., Koronakis, E., Luisi, B. and Hughes, C. (2000) Crystal structure of the bacterial membrane protein TolC central to multidrug efflux and protein export. Nature, 405, 914-919.

Lazdunski, C.J., Bouveret, E., Rigal, A., Journet, L., Lloubes, R. and Benedetti, H. (1998) Colicin import into Escherichia coli cells. J. Bacteriol., 180, 4993-5002.

Locher, K.P., Rees, B., Koebnik, R., Mitschler, A., Moulinier, L., Rosenbusch, J.P. and Moras, D. (1998) Transmembrane signaling across the ligand-gated FhuA receptor: crystal structures of free and ferrichromebound states reveal allosteric changes. Cell, 95, 771-778.

Nambu, T., Minamino, T., Macnab, R.M. and Kutsukake, K. (1999) Peptidoglycan-hydrolyzing activity of the FlgJ protein, essential for flagellar rod formation in Salmonella typhimurium. J. Bacteriol., 181, $1555-1561$.

Page, R.D.M. (1996) TREEVIEW: An application to display phylogenetic trees on personal computers. Comput. Appl. Biosci., 12, 357-358.

Paulsen, I.T., Park, J.H., Choi, P.S. and Saier, M.H., Jr (1997) A family of gram-negative bacterial outer membrane factors that function in the export of proteins, carbohydrates, drugs and heavy metals from gramnegative bacteria. FEMS Microbiol. Lett., 156, 1-8.
Pautsch, A. and Schulz, G.E. (1998) Structure of the outer membrane protein A transmembrane domain. Nature Struct. Biol., 5, 1013-1017.

Pils1, H. and Braun, V. (1995) Novel colicin 10: assignment of four domains to TonB- and TolC-dependent uptake via the Tsx receptor and to pore formation. Mol. Microbiol., 16, 57-67.

Russel, M. (1998) Macromolecular assembly and secretion across the bacterial cell envelope: type II protein secretion systems. J. Mol. Biol., 279, 485-499.

Saint, N., Lou, K.L., Widmer, C., Luckey, M., Schirmer, T. and Rosenbusch, J.P. (1996) Structural and functional characterization of OmpF porin mutants selected for larger pore size. II. Functional characterization. J. Biol. Chem., 271, 20676-20680.

Schirmer, T., Keller, T.A., Wang, Y.F. and Rosenbusch, J.P. (1995) Structural basis for sugar translocation through maltoporin channels at 3.1 ̊ resolution. Science, 267, 512-514.

Snijder, H.J., Ubarretxena-Belandia, I., Blaauw, M., Kalk, K.H., Verheij, H.M., Egmond, M.R., Dekker, N. and Dijkstra, B.W. (1999) Structural evidence for dimerization-regulated activation of an integral membrane phospholipase. Nature, 401, 717-721.

Song, L., Hobaugh, M.R., Shustak, C., Cheley, S., Bayley, H. and Gouaux, J.E. (1996) Structure of staphylococcal $\alpha$-hemolysin, a heptameric transmembrane pore. Science, 274, 1859-1866.

Thanabalu, T., Koronakis, E., Hughes, C. and Koronakis, V. (1998) Substrate-induced assembly of a contiguous channel for protein export from E.coli: reversible bridging of an inner-membrane translocase to an outer membrane exit pore. EMBO J., 17, 6487-6496.

Zgurskaya, H.I. and Nikaido, H. (2000) Multidrug resistance mechanisms: drug efflux across two membranes. Mol. Microbiol., 37, 219-225.

DOI: 10.1093/embo-reports/kvd075 